\title{
Influência da Imersão nas Respostas \\ Cardiorrespiratórias em Repouso
}

\section{Influence of Immersion on Resting Cardiorespiratory Responses}

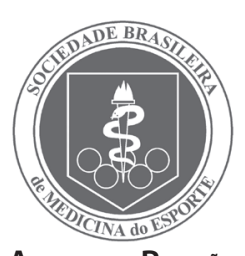

Artigo de ReVisão
Cristine Lima Alberton

Luiz Fernando Martins Kruel

Grupo de Pesquisa em Atividades Aquáticas e Terrestres, Escola de Educação Física. Universidade Federal do Rio Grande do Sul. Porto Alegre, Rio Grande do Sul, Brasil.

\section{Endereço para correspondência: Cristine Lima Alberton \\ Rua Felizardo, 750 - Jardim \\ Botânico \\ Laboratório de Pesquisa do \\ Exercício, sala 208 \\ 90690-200 - Porto Alegre, RS, Brasil E-mail: tinialberton@yahoo.com.br}

Submetido em 16/04/2008

Versão final recebida em 12/11/2008 Aceito em 07/12/2008

\begin{abstract}
RESUMO
Diversos benefícios nos componentes da aptidão física podem ser adquiridos com a prática de exercícios aquáticos. Além disso, a água proporciona um ambiente para a prática de exercícios com reduzido impacto nos membros inferiores e maior ou menor sobrecarga cardiorrespiratória, de acordo com os movimentos realizados. Porém, tais exercícios podem produzir respostas fisiológicas diferentes daquelas ao ar livre, visto que ocorrem alterações fisiológicas importantes durante a imersão, sendo importante a compreensão das mesmas em repouso para melhor prescrição nesse meio. O presente estudo tem como objetivo revisar estudos sobre o comportamento da frequência cardíaca e do consumo de oxigênio durante a imersão em repouso e compreender os fatores que influenciam nesse comportamento. Várias pesquisas indicam que a frequência cardíaca de repouso é reduzida com a imersão em meio aquático, porém, é importante salientar que fatores tais como temperatura da água, posição corporal, profundidade de imersão e frequência cardíaca inicial podem minimizar ou maximizar tais respostas. Os estudos que abordaram as respostas de consumo de oxigênio não são conclusivos; entretanto, ao contrário da frequência cardíaca, os mesmos indicam semelhante ou maior resposta durante a imersão em repouso. Assim, pode-se concluir que, devido às alterações cardiorrespiratórias verificadas com a imersão em ambiente aquático, a prescrição de exercícios nesse meio deve ser diferenciada daquela para exercícios em ambiente terrestre.
\end{abstract}

Palavras-chave: frequência cardíaca, consumo de oxigênio, meio aquático.

\begin{abstract}
Several benefits in the physical fitness components can be acquired with the practice of aquatic exercises. Moreover, water provides an environment for the practice of exercises with reduced impact on lower limbs and major or minor cardiorespiratory overload, depending on the movements performed. However, such exercises can produce physiological responses different from those on dry land, since important physiological alterations occur during immersion, being important to understand them at rest for better prescription in this environment. The present study has the purpose to review studies about the heart rate behavior and the oxygen uptake during immersion at rest and to understand the factors that influence in this behavior. Several investigations indicate that heart rate at rest is reduced with immersion in aquatic environment; however, it is important to highlight that factors such as water temperature, body position, immersion depth and initial heart rate may minimize or maximize such responses. The studies which approached the oxygen uptake responses are not conclusive; however, contrary to heart rate, the same ones indicate similar or greater response during immersion at rest. Thus, it can be concluded that, due to the verified cardiorespiratory alterations with immersion in aquatic environment, the prescription of exercises in this environment must be differentiated from exercises on dry land.
\end{abstract}

Keywords: heart rate, oxygen uptake, aquatic environment.

\section{INTRODUÇÃO}

A prática de exercícios aquáticos está sendo cada vez mais indicada, visto que têm sido comprovados seus diversos benefícios nos componentes da aptidão física. Entre tais benefícios estão as melhorias no condicionamento cardiorrespiratório ${ }^{(1-6)}$, na composição corporal(5,7), o aumento dos níveis de força ${ }^{(5,8,9)}$ e de flexibilidade ${ }^{(5,6,10)}$.

A vantagem da execução de exercícios aquáticos em relação aos terrestres é que, além de ser possível atingir benefícios nos diversos componentes da aptidão física, o meio líquido proporciona reduzido impacto nos membros inferiores ${ }^{(11-13)}$ e maior ou menor sobrecarga cardiorrespiratória, de acordo com os movimentos realizados ${ }^{(11,14-18)}$.
Todas as vantagens dos exercícios aquáticos estão associadas às características físicas da água. De acordo com Avellini et al.(1), pode-se esperar que o exercício físico aquático produza reações fisiológicas diferentes daquelas ao ar livre, devido tanto ao efeito hidrostático da água no sistema cardiorrespiratório, como sua capacidade de intensificar a perda de calor comparada com o ar. Logo, é importante a melhor compreensão dessas alterações fisiológicas em imersão em repouso para que seja possível a realização de melhor prescrição nesse meio.

O objetivo do presente estudo foi revisar o comportamento da frequência cardíaca e do consumo de oxigênio durante a imersão em repouso e compreender os fatores que influenciam nesse comportamento. 


\section{METODOLOGIA}

Para a realização da presente revisão, foram buscadas referências clássicas e atuais sobre o referido tema. Os artigos clássicos, dissertação e tese citados foram obtidos do banco de artigos do Grupo de Pesquisa em Atividades Aquáticas e Terrestres da UFRGS, e podem ser encontrados nas bibliotecas setoriais dessa universidade. Os artigos mais atuais foram retirados das bases de dados Scopus e Pubmed. Pela maior disponibilidade e facilidade na procura e na leitura, foram preferencialmente utilizados artigos publicados em língua portuguesa e inglesa. Para tal busca, palavras-chave como heart rate, oxygen uptake, immersion, aquatic environment, hydrogymnastics e water aerobics e suas respectivas traduções foram combinadas, entre outras.

\section{Comportamento da frequência cardíaca de repouso durante a imersão}

Já está bem consolidado na literatura que a frequência cardíaca (FC) de repouso sofre influência com a imersão no meio líquido. Entretanto, os estudos que analisaram o comportamento da FC em meio líquido apresentam dados bastante controversos, sendo encontrados valores de bradicardia, taquicardia ou nenhuma alteração.

Essa variação da FC de repouso no meio líquido depende da temperatura da água ${ }^{(19-23)}$, da posição corporal ${ }^{(24-26)}$, da profundidade de imersão(27-30), da FC inicial(28,30) e da redução do peso hidrostático ${ }^{(26)}$.

Arborelius et al. ${ }^{(31)}$ analisaram as respostas hemodinâmicas de homens em imersão na água até a profundidade de pescoço, em temperatura de $35^{\circ} \mathrm{C}$, considerada como termoneutra para repouso. Não foram observadas mudanças significativas na FC de repouso durante 30 min de imersão, embora tenha ocorrido tendência ao decréscimo quando comparado com o ambiente terrestre. Da mesma forma, Christie et al. ${ }^{(32)}$ avaliaram a FC de homens imersos com a cabeça para fora da água, em temperaturas de 34 a $34,5^{\circ} \mathrm{C}$, comparada com o meio terrestre. Não foram observadas diferenças estatisticamente significativas nessa variável entre os meios aquático e terrestre.

Ao contrário dos estudos anteriores, Watenpaugh et al. ${ }^{(33)}$ encontraram diminuição significativa na FC durante a imersão na água tanto para mulheres (21\%) como para homens (16\%) ao comparar o meio terrestre com a imersão na água na profundidade do pescoço, em temperatura de $34,6^{\circ} \mathrm{C}$ (similar à dos estudos supracitados). Já Nakanishi et al. ${ }^{(34)}$ também encontraram redução significativa de 4,7bpm na FC de repouso em imersão na água. Todos os indivíduos eram do sexo masculino e permaneceram com a cabeça para fora da água e o corpo em flutuação pelo uso de um cinturão flutuador comparado com a posição sentada em ambiente terrestre. Cabe salientar que a temperatura da água utilizada nesse estudo foi de $32,5^{\circ} \mathrm{C}$, abaixo da temperatura considerada como termoneutra para repouso, que foi utilizada nos estudos de Arborelius et al. ${ }^{(31)}$, Christie et al. ${ }^{(32)}$ e Watenpaugh et al. ${ }^{(33)}$ (34 a $35^{\circ} \mathrm{C}$ ).

As temperaturas de água utilizadas nos estudos supracitados variaram de 32,5 a $35^{\circ} \mathrm{C}$ e os resultados dessas pesquisas diferiram entre si. Outros estudos foram realizados, analisando e comparando as respostas hemodinâmicas em diferentes temperaturas de água. Craig e Dvorak ${ }^{(19)}$ observaram que a imersão na profundidade do queixo, em temperaturas de água de 36 e $37^{\circ} \mathrm{C}$, conduz a aumento significativo na FC de repouso, quando comparado com o meio terrestre. Em contrapartida, temperaturas iguais ou menores que $35^{\circ} \mathrm{C}$ no ambiente aquático resultaram em respostas de FC significativamente menores que no ambiente terrestre. Corroborando o estudo anterior, Rennie et al. ${ }^{(20)}$, que estudaram a imersão de indivíduos em repouso, durante o período de uma hora, na profundidade do queixo, verificaram que em temperaturas menores que $34^{\circ} \mathrm{C}$, a FC reduziu-se $25 \%$ abaixo dos valores do ambiente terrestre. Já na temperatura de $36^{\circ} \mathrm{C}$, os autores não observaram diferenças significativas entre os meios.
Park et al.(21) compararam os parâmetros cardiovasculares em homens em ambiente terrestre e em imersão na água em temperaturas de 34,5 e $30^{\circ} \mathrm{C}$, na profundidade de pescoço. Analisando-se a FC nas diferentes situações experimentais, foi observada redução significativa de $15 \%$ na situação de imersão em $30^{\circ} \mathrm{C}$ comparada com as outras situações. Graef et al. ${ }^{(23)}$ estudaram o comportamento da FC de repouso em indivíduos imersos em três diferentes condições de temperatura de água $\left(33,30\right.$ e $\left.27^{\circ} \mathrm{C}\right)$, na profundidade entre apêndice xifoide e ombros. De acordo com seus resultados, foi observada redução na FC em imersão aquática comparada com o meio terrestre em todas as temperaturas. Entretanto, diferenças estatisticamente significativas foram detectadas somente entre as médias de redução da FC nas temperaturas de $33^{\circ} \mathrm{C}$ $(17,85 \pm 10,67 \mathrm{bpm})$ e $27^{\circ} \mathrm{C}$ (33,75 \pm 11,27bpm), demonstrando assim diminuição mais acentuada da FC com a redução da temperatura da água. Além das temperaturas de água anteriormente testadas, Srámek et al. ${ }^{(22)}$ verificaram as respostas da FC de homens imersos com a cabeça para fora da água, durante uma hora, em temperaturas de água de 32, 20 e $14^{\circ} \mathrm{C}$. Em imersão a $32^{\circ} \mathrm{C}$ e $20^{\circ} \mathrm{C}$, a FC diminuiu significativamente $15 \%$ comparada com o ambiente terrestre. Já em $14^{\circ} \mathrm{C}$, a FC obteve aumento de 5\%. É importante considerar que nessa última temperatura, os indivíduos tiveram tremores, induzidos pelo frio, que podem ser considerados como exercícios leves e, consequentemente, aumentar a FC.

De forma geral, a partir dos resultados apresentados nos estudos anteriores que analisaram as respostas da FC em imersão em distintas temperaturas, podemos concluir que temperaturas de água maiores ou iguais a $36^{\circ} \mathrm{C}$ provocam aumento ou nenhuma alteração no comportamento da FC comparado com o meio terrestre. Já temperaturas menores que $34^{\circ} \mathrm{C}$ resultam em redução da $\mathrm{FC}$, excetuando-se temperaturas muito baixas, como, por exemplo, a temperatura de $14^{\circ} \mathrm{C}$ utilizada na pesquisa de Srámek et al.(22).

Além da temperatura da água, mudanças na posição corporal também podem refletir-se em alterações significativas nas respostas de FC. Analisando diferentes posições corporais, Connelly et al. ${ }^{(25)}$ verificaram que a FC de repouso não diferiu significativamente entre as situações de decúbito dorsal em ambiente terrestre, sentado em ambiente terrestre e sentado em imersão na água, após 45 min de imersão. A piscina foi mantida em temperatura entre 34,5 e $35^{\circ} \mathrm{C}$ e os indivíduos permaneceram imersos na profundidade de ombros. Respostas semelhantes foram encontradas por Sheldahl et al.(24), que analisaram as respostas da FC de homens nas posições de decúbito dorsal e ortostase no ambiente terrestre, e ortostase no ambiente aquático, em imersão na profundidade dos ombros, com a temperatura da água mantida em $31^{\circ} \mathrm{C}$, e não observaram diferenças estatisticamente significativas entre as três situações.

Ao contrário dos estudos anteriores, Kruel et al. ${ }^{(26)}$ analisaram as respostas da FC de homens imersos em meio líquido na profundidade de cicatriz umbilical, em temperatura de $30^{\circ} \mathrm{C}$, comparado com o ambiente terrestre nas posições de decúbito dorsal e ortostase. Como resultados, foi encontrada redução significativa de $20 \%$ para as posições de decúbito dorsal no ambiente terrestre e ortostase no ambiente aquático comparado com a posição ortostática em ambiente terrestre. Esses resultados demonstram que a imersão em ortostase nessa temperatura de água pode reduzir a FC para valores semelhantes aos da posição de decúbito dorsal pelo retorno venoso facilitado.

Outro fator que também pode exercer influência na FC é a profundidade de imersão. Tratando-se de diferentes profundidades, Gleim e Nicholas ${ }^{(27)}$ imergiram indivíduos no meio aquático em temperatura de água mantida a $30,5^{\circ} \mathrm{C}$ e compararam as respostas de FC com o ambiente terrestre. Nenhuma diferença significativa foi encontrada para a FC de repouso entre as diferentes profundidades de imersão testadas (maléolo, patela, coxa e cicatriz umbilical). 
Não obstante, outros estudos encontraram diferenças na FC em imersão entre diferentes profundidades, com a análise do comportamento dessa variável também em profundidades maiores que cicatriz umbilical. Kruel(28) analisou o comportamento da FC em indivíduos jovens, de ambos os sexos, durante imersão vertical em repouso nas profundidades de imersão de tornozelo, joelho, quadril, cicatriz umbilical, processo xifoide, ombros, pescoço e ombros com braços para fora da água. A temperatura da água foi mantida entre 29 e $30^{\circ} \mathrm{C}$. Foram encontradas diferenças significativas a partir do quadril, com redução na FC de 10,6\%, chegando a 18,7\% na profundidade do pescoço. A pesquisa de Kruel et al. ${ }^{(29)}$ demonstrou resultados similares aos do estudo anterior, ao verificar as alterações da FC impostas por diferentes profundidades de imersão vertical em repouso. Nesse estudo, o $n$ amostral $(n=177)$ e a variação de idade foram maiores (18 a 65 anos), com a análise de indivíduos de ambos os sexos. A temperatura utilizada foi de 29 a $30^{\circ} \mathrm{C}$. Resultados similares foram encontrados, sem diferenças significativas entre os sexos e as diferentes faixas etárias, e com redução significativa de 9,4\% na FC a partir do quadril até 17,6\% na profundidade do pescoço.

Coertjens et al. ${ }^{(30)}$ analisaram 395 indivíduos, de ambos os sexos, durante a imersão vertical em repouso nas mesmas profundidades e temperatura que o estudo de Kruel(28). A faixa etária nesse estudo foi mais abrangente, sendo analisados indivíduos de sete a 75 anos. Não foram observadas diferenças significativas entre as faixas etárias e os sexos. Entretanto, também foram encontradas diferenças significativas a partir da profundidade do quadril, com redução de 9,1\%. Nesse estudo, outro importante resultado observado foi que, quanto maior a FC inicial, maior a redução da FC em imersão. Para valores de FC iniciais de 40 a 49bpm, redução média de 1 bpm foi encontrada, enquanto que para valores de FC iniciais entre 130 e 139bpm, redução média de 32,67bpm pode ser observada. Os autores constataram que, além da profundidade de imersão, a FC inicial avaliada fora da água também exerce influência na resposta da FC.

De acordo com esses resultados, podemos concluir que a profundidade de imersão exerce influência na FC, visto que, quanto mais imerso está o corpo, maior é a ação da pressão hidrostática. Com a ação da pressão hidrostática mais acentuada, maior pode ser a redução da FC, quando a temperatura da água e a posição corporal são devidamente controladas. Além disso, a FC inicial dos sujeitos também é de fundamental importância na magnitude dessa redução.

Esse fato ocorre porque a pressão hidrostática é um dos principais fatores que influenciam na FC em imersão ${ }^{(1,24,25,31-33)}$. Com a imersão, ocorre aumento no volume sanguíneo central, devido à redistribuição do sangue venoso e fluido extracelular dos membros inferiores para a região central. Com o aumento do volume plasmático na região central, o coração e os vasos da circulação central são distendidos, gerando estimulação nos receptores de volume e pressão desses tecidos. Isso conduz à readaptação no sistema cardiovascular, aumentando a pressão venosa central, o débito cardíaco e o volume sistólico, para, enfim, diminuir a $\mathrm{FC}^{(33)}$

Provavelmente, as condições térmicas oferecidas pelo meio aquático representam também um dos mecanismos responsáveis pela redução da FC em imersão, devido à facilitação da troca de calor entre o organismo e o meio ambiente. Por causa disso, a necessidade de distribuir sangue da região central (tórax e abdômen) para a periferia é diminuída, fazendo com que o volume plasmático se concentre na região central, acrescentando mais um fator para estimular os receptores de volume e de pressão do coração e sistema vascular central|(19).
De acordo com os resultados de Kruel et al.(26), outro possível mecanismo responsável pela redução da FC advinda da imersão no meio aquático esteja relacionado com o comportamento de diminuição do peso hidrostático oferecido por esse meio. Os autores concluíram que, com a redução do peso hidrostático, provavelmente seja necessário menor recrutamento muscular para manter a postura em pé, diminuindo a necessidade de aporte sanguíneo para os membros inferiores, auxiliando também na concentração de sangue na região central do organismo.

A partir das explicações acima, pode-se concluir que a associação desses três mecanismos principais (a pressão hidrostática, os efeitos térmicos da água e a redução do peso hidrostático) é responsável pelas alterações hemodinâmicas decorrentes da imersão. Considerando a queda da FC em imersão, Sovaa ${ }^{(35)}$ propôs uma equação para a prescrição de aulas em meio aquático com base no comportamento da $F C: F C_{\text {máx }}$ água $=F C_{\text {máx }}$ terra -17 . Cabe salientar que, nessa proposta, a autora considera um fator de correção para o meio aquático fixo (redução de 17bpm) para todos os indivíduos. Todavia, alguns fatores, tais como a temperatura da água, a posição corporal, a profundidade de imersão e a FC inicial do indivíduo, podem maximizar ou minimizar o comportamento da FC em imersão. Nesse sentido, a proposta atual de Graef e Kruel(36) parece ser mais adequada, uma vez que leva em consideração um fator de correção individual: $F C_{\text {máx }}$ água $=F_{\text {máx }}$ terra - $\Delta \mathrm{FC}$, onde $\Delta \mathrm{FC}$ corresponde à medida da redução da $F C$ decorrente da imersão na profundidade, temperatura da água e posição corporal utilizadas para o exercício.

No quadro 1 são apresentados os resumos dos estudos supracitados, com as respectivas temperaturas e profundidades de imersão utilizadas e respostas de frequência cardíaca de repouso em imersão.

\section{Comportamento do consumo de oxigênio de repouso durante a imersão}

Existe certa divergência nas respostas do consumo de oxigênio $\left(\mathrm{VO}_{2}\right)$ de repouso em imersão. Enquanto alguns autores encontraram aumento significativo no $\mathrm{VO}_{2}{ }^{(37-39)}$, outros não verficaram diferenças significativas ${ }^{(21,24,27,32,38)}$

Estudos de Christie et al. ${ }^{(32)}$ e Park et al. ${ }^{(21)}$ não encontraram diferenças significativas no $\mathrm{VO}_{2}$ de indivíduos entre os meios terrestre e aquático, sendo a profundidade de imersão no pescoço e a temperatura da água mantida em $34,5^{\circ} \mathrm{C}$, considerada como termoneutra para repouso. Vale ressaltar que ambos os estudos realizaram as medidas de repouso com os indivíduos sentados em um cicloergômetro, possibilitando, assim, menor recrutamento de fibras musculares para a manutenção da postura em pé, além do reduzido peso hidrostático oferecido pelo meio aquático.

Corroborando os estudos anteriores, Johnson et al.(40) não encontraram diferenças significativas no $\mathrm{VO}_{2}$ de indivíduos imersos na profundidade dos ombros em temperaturas de água mantidas entre 26 e $26,5^{\circ} \mathrm{C}$. Entretanto, foi observada tendência de maiores valores para o repouso em pé no meio aquático comparado com o meio terrestre, tanto para homens (aumento de $0,57 \mathrm{ml}_{*} \mathrm{~kg}^{-1} * \mathrm{~min}^{-1}$ ) como para mulheres (aumento de $0,51 \mathrm{ml}_{*} \mathrm{~kg}^{-1} \mathrm{~min}^{-1}$ ).

Já o estudo de Gleim e Nicholas(27) analisou o $\mathrm{VO}_{2}$ de repouso em indivíduos, de ambos os sexos, imersos na água em temperatura de $30,5^{\circ} \mathrm{C}$ em diferentes profundidades de imersão. Não foram encontradas diferenças significativas no $\mathrm{VO}_{2}$ entre a posição ortostática fora da água e as profundidades de imersão de maléolo, patela, coxa e cicatriz umbilical, medidas essas que foram avaliadas em dias diferentes. Entretanto, nesse estudo, os indivíduos não foram avaliados em maiores profundidades de imersão, nas quais a ação da pressão hidrostática é mais acentuada. 
Quadro 1. Comportamento da frequência cardíaca $(F C)$ durante a imersão em repouso comparado com o meio terrestre

\begin{tabular}{|c|c|c|c|}
\hline Autor & Temperatura da água & Profundidade de imersão & Resultados \\
\hline Arborelius et al. ${ }^{(31)}$ & $35^{\circ} \mathrm{C}$ & Pescoço & Sem mudanças significativas na FC \\
\hline Sheldahl et al.(24) & $31{ }^{\circ} \mathrm{C}$ & Ombros & Sem mudanças significativas na FC \\
\hline Christie et al. (32) & Entre 34 e $34,5^{\circ} \mathrm{C}$ & Pescoço & Sem mudanças significativas na FC \\
\hline Connelly et al..(25) & Entre 34 e $34,5^{\circ} \mathrm{C}$ & Ombros & Sem mudanças significativas na FC \\
\hline Nakanishi et al.(34) & $32,5^{\circ} \mathrm{C}$ & Pescoço sem apoio dos pés & Redução significativa de 4,7bpm na FC \\
\hline Watenpaugh et al.(33) & $34,6^{\circ} \mathrm{C}$ & Pescoço & Redução significativa na FC de 21\% para mulheres e 16\% para homens \\
\hline Kruel et al.(26) & $30^{\circ} \mathrm{C}$ & Cicatriz umbilical & Redução significativa de $20 \%$ na FC \\
\hline Craig e Dvorak $k^{(19)}$ & Diferentes temperaturas & Queixo & $\begin{array}{l}\text { Aumento significativo na } \mathrm{FC} \text { para temperaturas de } 36 \text { e } 37^{\circ} \mathrm{C} \text { e redução } \\
\text { significativa para temperaturas iguais ou menores que } 35^{\circ} \mathrm{C}\end{array}$ \\
\hline Rennie et al.(20) & Diferentes temperaturas & Queixo & $\begin{array}{l}\text { Sem mudanças significativas na FC para a temperatura de } 36^{\circ} \mathrm{C} \text { e redução } \\
\text { significativa de } 25 \% \text { para temperaturas iguais ou menores que } 34^{\circ} \mathrm{C}\end{array}$ \\
\hline Park et al..(21) & 34,5 e $30^{\circ} \mathrm{C}$ & Pescoço & $\begin{array}{l}\text { Sem mudanças significativas na } \mathrm{FC} \text { para a temperatura de } 34,5^{\circ} \mathrm{C} \text { e redu- } \\
\text { ção significativa de } 15 \% \text { para a temperatura de } 30^{\circ} \mathrm{C}\end{array}$ \\
\hline Srámek et al. ${ }^{(22)}$ & 32,20 e $14^{\circ} \mathrm{C}$ & Pescoço & $\begin{array}{l}\text { Redução significativa na FC para temperaturas de } 32 \text { e } 20^{\circ} \mathrm{C} \text { e aumento } \\
\text { significativo para a temperatura de } 14^{\circ} \mathrm{C}\end{array}$ \\
\hline Graef et al. (23) & 33,30 e $27^{\circ} \mathrm{C}$ & Entre processo xifoide e ombros & $\begin{array}{l}\text { Redução significativa na FC para as três temperaturas; a resposta de } \mathrm{FC} \\
\text { em } 27^{\circ} \mathrm{C} \text { foi significativamente menor que em } 33^{\circ} \mathrm{C}\end{array}$ \\
\hline Gleim e Nicholas (27) & $30,5^{\circ} \mathrm{C}$ & Maléolo, patela, coxa e cicatriz umbilical & $\begin{array}{l}\text { Sem mudanças significativas na FC para nenhuma das profundidades } \\
\text { de imersão }\end{array}$ \\
\hline Kruel(28) $^{(28)}$ & Entre 29 e $30^{\circ} \mathrm{C}$ & $\begin{array}{l}\text { Tornozelo, joelho, quadril, cicatriz umbili- } \\
\text { cal, processo xifoide, ombros e pescoço }\end{array}$ & $\begin{array}{l}\text { Redução significativa de } 10,6 \% \text { na FC na profundidade do quadril, atin- } \\
\text { gindo } 18,7 \% \text { na profundidade do pescoço }\end{array}$ \\
\hline Kruel et al.(29) & Entre 29 e $30^{\circ} \mathrm{C}$ & $\begin{array}{l}\text { Tornozelo, joelho, quadril, cicatriz umbili- } \\
\text { cal, processo xifoide, ombros e pescoço }\end{array}$ & $\begin{array}{l}\text { Redução significativa de } 9,4 \% \text { na FC na profundidade do quadril, atingin- } \\
\text { do } 17,6 \% \text { na profundidade do pescoço }\end{array}$ \\
\hline Coertjens et al..$^{(30)}$ & Entre 29 e $30^{\circ} \mathrm{C}$ & $\begin{array}{l}\text { Tornozelo, joelho, quadril, cicatriz umbili- } \\
\text { cal, processo xifoide, ombros e pescoço }\end{array}$ & $\begin{array}{l}\text { Redução significativa de } 9,1 \% \text { na FC na profundidade do quadril, atingin- } \\
\text { do } 15,3 \% \text { na profundidade do pescoço }\end{array}$ \\
\hline
\end{tabular}

Outros estudos avaliaram o comportamento do $\mathrm{VO}_{2}$ de repouso em diferentes posições corporais. Sheldahl et al.(24) avaliaram homens jovens nas posições de decúbito dorsal e ortostase fora da água e ortostase em imersão na profundidade dos ombros em temperatura de $31^{\circ} \mathrm{C}$. Não foram observadas diferenças significativas no $\mathrm{VO}_{2}$ entre as três situações.

Kruel et al. ${ }^{(39)}$ analisaram o comportamento do $\mathrm{VO}_{2}$ de mulheres fisicamente ativas fora da água e em imersão em temperatura de $32^{\circ} \mathrm{Ce}$ profundidade de processo xifoide nas mesmas posições de Sheldahl et al.(24). Entretanto, os autores observaram tendência de aumento no $\mathrm{VO}_{2}$ da posição decúbito dorsal fora da água $\left(3,17 \pm 0,34 \mathrm{~m}_{*} \mathrm{~kg}^{-1} * \mathrm{~min}^{-1}\right)$ para posição ortostática fora da água $\left(3,62 \pm 0,36 \mathrm{~m}_{*} \mathrm{~kg}^{-1} * \mathrm{~min}^{-1}\right)$ e dessa para a posição ortostática em imersão $\left(3,98 \pm 0,44 \mathrm{ml}^{*} \mathrm{~kg}^{-1} * \mathrm{~min}^{-1}\right)$, com diferenças significativas somente entre decúbito dorsal e imersão (aumento de 25,5\%). Fatores tais como a diminuição da frequência cardíaca e o menor recrutamento de fibras musculares devido à redução do peso hidrostático sugerem que as posições de decúbito dorsal e imersão sejam fisiologicamente mais semelhantes do que as posições ortostática fora da água e ortostática em imersão. Dessa forma, a imersão provocou aumento significativo do $\mathrm{VO}_{2} \mathrm{com}$ a associação da mudança de posição corporal.

Em contrapartida, estudos com temperaturas de áqua fria encontraram aumento no $\mathrm{VO}_{2}$ de repouso em imersão. Mekjavick e Bli$\mathrm{gh}^{(38)}$ analisaram a resposta do $\mathrm{VO}_{2}$ em imersão na profundidade do manúbrio em temperaturas de água de 10,15 e 20 $\mathrm{C}$ e encontraram aumentos significativos para todas as temperaturas quando comparadas com os níveis de pré-imersão, sem diferenças significativas entre elas. McArdle et al.(37) também encontraram $\mathrm{VO}_{2}$ de repouso significativamente maior em imersão em temperatura de $18^{\circ} \mathrm{C}$ comparado com $33^{\circ} \mathrm{C}$. Isso pode ser explicado pelo fato de que na água fria há diminuição da temperatura da pele, juntamente com respiração ofegante do indivíduo, aumentando assim o consumo de oxigênio no músculo respiratório, que pode representar uma parte do aumento do $\mathrm{VO}_{2}$ gera|(38).

Porém, o estudo de Mekjavik e Bligh ${ }^{(38)}$ também analisou o $\mathrm{VO}_{2}$ em imersão em água quente, em temperatura de $40^{\circ} \mathrm{C}$. Da mesma forma, nessa temperatura foi observado aumento significativo do $\mathrm{VO}_{2}$; isso demonstra que o comportamento do $\mathrm{VO}_{2}$ não depende só da temperatura fria da água.

Um dos principais fatores responsáveis por esse aumento do $\mathrm{VO}_{2}$ em imersão em repouso é a pressão hidrostática. Sua ação em diferentes partes do corpo, principalmente nos membros inferiores e abdômen, proporciona redistribuição sanguínea, com consequente aumento do retorno venoso e concentração do volume sanguíneo na região central. Com o aumento da passagem de sangue pelo coração e pulmões, há também maior captação de oxigênio pelo sangue, proporcionando aumento no $\mathrm{VO}_{2}{ }^{(38)}$.

No quadro 2 são apresentados os resumos dos estudos supracitados, com as respectivas temperaturas e profundidades de imersão utilizadas e respostas de consumo de oxigênio de repouso em imersão. 
Quadro 2. Comportamento do consumo de oxigênio $\left(\mathrm{VO}_{2}\right)$ durante a imersão em repouso comparado com o meio terrestre

\begin{tabular}{|c|c|c|c|}
\hline Autor & Temperatura da água & Profundidade de imersão & Resultados \\
\hline Johnson et al. ${ }^{(40)}$ & Entre 26 e $26,5^{\circ} \mathrm{C}$ & Ombros & Sem mudanças significativas no $\mathrm{VO}_{2}$ \\
\hline McArdle et al. ${ }^{(37)}$ & 33,25 e $18^{\circ} \mathrm{C}$ & Primeira vértebra torácica & $\begin{array}{l}\text { Sem mudanças significativas no } \mathrm{VO}_{2} \text { para as temperaturas de } 33 \text { e } 25^{\circ} \mathrm{C} \text { e aumento } \\
\text { significativo para a temperatura de } 18^{\circ} \mathrm{C}\end{array}$ \\
\hline Sheldahl et al.(24) & $31{ }^{\circ} \mathrm{C}$ & Ombros & Sem mudanças significativas no $\mathrm{VO}_{2}$ \\
\hline Gleim e Nicholas ${ }^{(27)}$ & $30,5^{\circ} \mathrm{C}$ & Maléolo, patela, coxa e cicatriz umbilical & Sem mudanças significativas no $\mathrm{VO}_{2}$ para nenhuma das profundidades de imersão \\
\hline Mekjavick e Bligh ${ }^{(38)}$ & $40,20,15$ e $10^{\circ} \mathrm{C}$ & Manúbrio & Aumento significativo no $\mathrm{VO}_{2}$ para todas as temperaturas de água \\
\hline Christie et al. ${ }^{(32)}$ & Entre 34 e $34,5^{\circ} \mathrm{C}$ & Pescoço & Sem mudanças significativas no $\mathrm{VO}_{2}$ \\
\hline Park et al. ${ }^{(21)}$ & 34,5 e $30^{\circ} \mathrm{C}$ & Pescoço & Sem mudanças significativas no $\mathrm{VO}_{2}$ \\
\hline Kruel et al. ${ }^{(39)}$ & $32^{\circ} \mathrm{C}$ & Processo xifoide & Sem mudanças significativas no $\mathrm{VO}_{2}$ \\
\hline
\end{tabular}

\section{CONSIDERAÇÕES FINAIS}

Como pode ser observado nos estudos supracitados, durante a imersão no meio aquático os indivíduos apresentam comportamento fisiológico diferente do do meio terrestre. É importante salientar que, devido a essas alterações cardiorrespiratórias decorrentes da imersão, a prescrição de exercícios nesse meio não deve ser a mesma que para exercícios em ambiente terrestre. Logo, para uma prescrição mais adequada é necessária a realização de testes máximos dentro da água, com medidas diretas de $\mathrm{VO}_{2}$ e FC. Quando isso não é possível, torna-se ne- cessária a utilização de equações de predição da FC máxima dentro da água, a partir de fatores de correção. Nesse sentido, sugerimos a escolha de equações com fatores de correção individuais, como a proposta por Graef e Kruel(36) $\left(F C_{\text {máx }}\right.$ água $=F C_{\text {máx }}$ terra $\left.-\Delta F C\right)$, e não com fatores de correção fixos, como na equação proposta por Sova ${ }^{(35)}\left(F C_{\text {máx }}\right.$ água = $F C_{\text {máx }}$ terra - 17), visto que a mesma não considera a variação da FC.

Todos os autores declararam não haver qualquer potencial conflito de interesses referente a este artigo.

\section{REFERÊNCIAS BIBLIOGRÁFICAS}

1. Avellini BA, Shapiro Y, Pandolf KB. Cardio-respiratory physical training in water and on land. Eur J Appl Physiol 1983;50:255-63.

2. Michaud TJ, Brennan DK, Wilder RP, Sherman NW. Aquarunning and gains in cardiorespiratory fitness. J Strength Cond Res 1995;9:78-84.

3. Taunton JE, Rhodes EC, Wolski LA, Donelly M, Warren J, Elliot J, et al. Effect of land-based and water-based fitness programs on the cardiovascular fitness, strenght and flexibility of woman aged 65-75 years. Gerontology 1996;42:204-10.

4. Davidson $K, M c$ Naughton L. Deep water running and road training improve $\mathrm{VO}_{2 \max }$ in untrained women. J Strength Cond Res 2000;14:191-5.

5. Takeshima N, Rogers ME, Watanabe WF, BrechueWF, Okada A, Yamada T, et al. Water-based exercise improves health-related aspects of fitness in older women. Med Sci Sports Exerc 2002;33:544-51.

6. Alves RV, Mota J, Costa MC, Alves JGB. Physical fitness and elderly health effects of hydrogymnastics. Rev Bras Med Esporte 2004;10:38-43

7. Gappmaier E, LakeW, Nelson AG, Fisher AG. Aerobic exercise in water versus walking on land: Effects on indices of fat reduction and weight loss of obese women. J Sports Med Phys Fitness 2006;46:564-9.

8. Pöyhönen T, Sipilä S, Keskinen KL, Hautala A, Savolainen J, Älkiä E. Effects of aquatic resistance training on neuromuscular performance in healthy women. Med Sci Sports Exerc 2002;34:2103-9.

9. Cardoso AS, Tartaruga LAP, Barella RE, Brentano MA, Kruel LFM. Effects of a deep water training program on women's muscle strenght. FIEP Bulletin 2004;74:590-3.

10. Yazawa RH, Rivet RE, França NM, Souza MT. Antropometria e flexibilidade em senhoras praticantes de ginástica aquática. Rev Bras Cienc Movim 1989;3:23-9.

11. Kruel LFM. Alterações fisiológicas e biomecânicas em indivíduos praticando exercícios de hidroginástica dentro e fora d'água [tese de doutorado]. Santa Maria (RS): Universidade Federal de Santa Maria; 2000. 111 p. Brasil.

12. Miyoshi T, Shirota T, Yamamoto S, Nakazawa K, Akai M. Effect of the walking speed to the lower limb joint angular displacements, joint moments and ground reaction forces during walking in water. Disabil Rehabil 2004;26:724-32.

13. Barela AMF, Stolf SF, Duarte M. Biomechanical characteristics of adults walking in shallow water and on land. J Electromyogr Kinesiol 2006;16:250-6.

14. Heberlein T, Perez HR, Wygand J, Connor K. The metabolic cost of high impact aerobic and hydroaerobic exercise in middle-aged females. Med Sci Sports Exerc 1987;19:589.

15. Cassady SL, Nielsen DH. Cardiorespiratory responses of healthy subjects to calisthenics performed on land versus in water. Phys Ther 1992;72:532-8.

16. Heithold K, Glass SC. Variations in the heart rate and perception of effort during land and water aerobics in older women. J Exerc Physiol 2002;5:22-8

17. Alberton CL, Coertjens M, Figueiredo PAP, Kruel LFM. Behavior of oxygen uptake in water exercises performed at different cadences in ad out of water. Med Sci Sports Exerc 2005;37:S103.

18. Alberton, CL, Olkoski MM, Pinto SS, Becker ME, Kruel LFM. Cardiorespiratory responses of postmenopausal women to different water exercises. Int J Aquat Res Educ 2007;1:363-72.

19. Craig AB, Dvorak M. Thermal regulation during water immersion. J Appl Physiol 1966;21:1577-85.

20. Rennie DW, Prampero P, Cerretelli P. Effects of water immersion on cardiac output, heart rate, and stroke volume of man at rest and during exercise. Med Dello Sport 1971;24:223-8.
21. Park KS, Choi JK, Park YS. Cardiovascular regulation during water immersion. J Physiol Appl Human Sci 1999;18:233-41.

22. Srámek P, Simecková M, Janski L, Savlíková J, Vybiral S. Human Physiological responses to immersion into water of different temperatures. Eur J Appl Physiol 2000;81:436-42.

23. Graef Fl, Tartaruga LAP, Alberton CL, Kruel LFM. Freqüência cardíaca em homens imersos em diferentes temperaturas de água. Rev Port Cienc Desp 2005;5:266-73.

24. Sheldahl LM, Wann LS, Clifford OS, Tristani FE, Wolf LG, Kalbfleish JH. Effect of central hypervolemia on cardiac performance during exercise. J Appl Physiol 1984;52:1662-7.

25. Connely TP, SheldahI LM, Tristani FE, Levandosky SG, KalkhoffRK, Hoffman MD, et al. Effect of increased central blood volume with water immersion on plasma catecholamines during exercise. J Appl Physiol 1990;69:651-6.

26. Kruel LFM, Tartaruga LAP, Alberton CL, Turra NA, Müller FG, Petkowicz R. Effects of hydrostatic weight on heart rate during immersion in water. Aquatic Fitness Research Journal 2004;1:4.

27. Gleim GW, Nicholas JA. Metabolic costs and heart rate responses to treadmill walking in water at different depths and temperatures. Am J Sports Med 1989;17:248-52

28. Kruel LFM. Peso hidrostático e freqüência cardíaca em pessoas submetidas a diferentes profundidades de água [dissertação de mestrado]. Santa Maria (RS): Universidade Federal de Santa Maria; 1994. 116 p. Brasil.

29. Kruel LFM, Tartaruga LAP, Dias AC, Silva RC, Picanço PSP, Rangel AB. Freqüência cardíaca durante imersão no meio aquático. Fit Perform J 2002;1:46-51.

30. Coertjens M, Dias ABC, Silva RC, Rangel ACB, Tartaruga LAP, Kruel LFM. Determinação da bradicardia durante imersão vertical no meio líquido. In: Anais do 12 Salão de Iniciaçăo Científica; 2000 Set 11-15; Porto Alegre, Brasil. Porto Alegre: Universidade Federal do Rio Grande do Sul; 2000. p. 341.

31. Arborelius M, Baldlin UI, Lilja B, Lundgren CEG. Hemodynamic changes in man during immersion with the head above water. Aerospace Med 1972;43:590-8

32. Christie JL, Sheldal LM, Tristani FE, Wann LS, Sagar KB, Levandoski SG, et al. Cardiovascular regulation during head-out water immersion exercise. J Appl Physiol 1990;69:657-64.

33. Watenpaugh DE, Pump B, Bie P, Norsk P. Does gender influence human cardiovascular and renal responses to water immersion? J Appl Physiol 2000;89:621-8.

34. Nakanishi Y, Kimura T, Yoko Y. Physiological responses to maximal treadmill and deep water running in the young and the middle aged males. Appl Human Sci 1999;18:31-5.

35. Sova R. Aquatics: The complete reference guide for aquatic fitness professionals. Boston: Jones \& Bartlett Publishers, 1992.

36. Graef FI, Kruel LFM. Freqüência cardíaca e percepção subjetiva do esforço no meio aquático: diferenças em relação ao meio terrestre e aplicaçõos na prescrição do exercício - uma revisão. Rev Bras Med Esporte 2006;12:221-8.

37. McArdle WD, Magel JR, Lesmes GR, Pechar GS. Metabolic and cardiovascular adjustment to work in air and water at 18,25 and $33^{\circ} \mathrm{C}$. J Appl Physiol 1976;40:85-90.

38. Mekjavick IB, Bligh J. The increased oxygen uptake upon immersion. Eur J Appl Physiol 1989;58:556-62.

39. Kruel LFM, Coertjens M, Pinto SS, Alberton CL, Brentano MA. Efeito da imersão sobre o comportamento do consumo de oxigênio em repouso. Revista Brasileira de Atividade Física \& Saúde 2006;11:25-31.

40. Johnson BL, Stromme SB, Adamczyk JW, Tennoe KO. Comparison of oxygen uptake and heart rate during exercises on land and in water. Phys Therapy 1977;57:273-8. 